\title{
A Preliminary Study on the Preparation of Monoclonal Antibody with Umbilical Cord Blood Cell
}

\author{
Dongmei Su', Shuren Zhang2, Shuzhi Zhang2, Ying Gong1, Shaohui Zhu², Liyan Zhang2, \\ Youjuan Wang ${ }^{2}$, Changqing Yue ${ }^{1}$, Xiaoping Liu ${ }^{1}$ \\ ${ }^{1}$ Department of Microbiology, Medical College, Qingdao University, Qingdao, China \\ ${ }^{2}$ Qingdao Zhongren Biology Technology Co. Ltd., Qingdao, China \\ Email: Sdongmei@126.com, zhongrenshengwu@163.com
}

Received January 2015

\begin{abstract}
Objective: We conducted a preliminary study on the feasibility of preparing monoclonal antibody by umbilical cord blood cells systems. Methods: Collected umbilical cord blood cells and stimulated by Rubella virus, then incubated them and collected cell supernatant. By using Caprylic acidsaturated Ammonium sulfate method, monoclonal antibody was purified, and IgG subtype identification was conducted by their subtype classification kit of sigma. In traditional way, the preparation of monoclonal antibody cannot do without BABL/C mice and SP2/0 hybridoma cells. Consider this kinds of monoclonal antibody as a positive control, the reactivity and specificity of monoclonal antibodies were identified by Dot-ELISA. Results: By ELISA, we obtained four strains of positive umbilical cord blood cells. After subculture, cryopreservation and resuscitation in vitro, three of them were confirmed to secrete monoclonal antibody against rubella virus stably. The result of monoclonal antibody subtype classification showed that, both IgG1 and IgG2 types were detected. However, the quantity of monoclonal antibody prepared by umbilical cord blood cells was less than that from traditional method. Conclusions: The method of prepare monoclonal antibody using umbilical cord blood cells is feasible.
\end{abstract}

\section{Keywords}

Umbilical Cord Blood Cells, Monoclonal Antibody, Rubella Virus

\section{Introduction}

Umbilical cord blood contains rich hematopoietic progenitor cells (hematopoietic progenitor cells, HPC), which contains a considerable number of hematopoietic progenitor cells or even more than bone marrow. Therefore, umbilical cord blood has attracted more and more attention in science, and shown a potential to replace bone marrow to become a new source of hematopoietic progenitor cells [1]. Researches about human umbilical cord blood mesenchymal stem cells (human umbilical cord blood mesenchymal stem cells, HUCB-MSCS) begin in 
the early part of the century, and then widely concerned by scientists. The basic composition of umbilical cord blood is the same with that of bone marrow. Rubinstein confirmed that, the colony formed by umbilical cord blood samples was larger than the formation of bone marrow, and umbilical cord blood might have a strong growth potential [2]. Back in 1984, Gabutti et al. found that cord blood contains blood stem cells and more colony forming cells, the ratio was significantly higher than that of peripheral blood, close to or higher than the adult bone marrow, containing about $2 \times 10^{6}$ myeloid progenitor cells and $1 \times 10^{6}$ erythroid progenitor cells in cord blood every $100 \mathrm{ml}$, this number is sufficiently heavy building blood function [3]. Umbilical cord blood is rich in stem cells. And stem cells are functional cells capable of multilineage differentiation, also known as the "universal cell”, can differentiate into various cells [4]-[7], including B cells. B cell can be activated, proliferate and differentiate into plasma cell after stimulated again by an antigen. Antigen stimulation can cause plasma cell activation and producing corresponding Immue globulin, and the relative content of IgG is $65 \%-80 \%$. IgG can combine with the corresponding specific antigen, and perform humoral immune function [8] [9]. In addition, umbilical cord blood is a rich source of, relatively "young", poor antigen presentation. Based on the above characteristics of umbilical cord blood, the author has preliminarily studied on preparation of monoclonal antibody for extraction of umbilical cord blood cells.

\section{Materials and Methods}

\subsection{Materials}

10 cord blood units were collected from the health maternal of department of gynecology and obstetrics of Affiliated Hospital of Qingdao University, and the maternal already discharged HIV, HBV, HCV, CMV, EBV, syphilis infection. Hydroxyethyl starch (HES) is American Dupont Pharma products. Monoclonal antibody subtype identification kit, horseradish peroxidase, Goat anti rat IgG-HRP were purchased from Sigma company. ELISA microplate was purchased from Costar Company USA. DAB chromogenic substrate solution was purchased from Tiangen Biotech (Beijing) Co., Ltd. Other routine reagents are all pure analysis and made in China. Monoclonal antibody standard were prepared by traditional method through the BABL/C mice and SP2/0 hybridoma cells, were preparated and provided by Affiliated Hospital of Qingdao University.

\subsection{Methods}

\subsubsection{Umbilical Cord Blood Collection}

With the closed blood sampling (sterile disposable plastic blood bag sampling method) [10], we collected 10 copies of umbilical cord blood without acute and chronic diseases from full-term parturients, who are in the maternity hospital affiliated to Qingdao University. Immediately umbilical cord is cut after the delivery of fetus, and the place of umbilical vein where we would be punctured disinfected with tincture of iodine. Use with 200 $\mathrm{ml}$ sterile blood bag $28 \mathrm{ml}$ ACD preservation solution, umbilical vein puncture, the umbilical cord blood into the blood bag by gravity. From time to time to shake the blood bag, it was sured that the umbilical cord blood and preservation solution mixed fully.

\subsubsection{Nucleated Cell Separation and Cell Viability Determination}

Hydroxyethyl starch (HES) sedimentation method was used to separate umbilical cord blood cells [11]. Calculating the recovery rate of NC use the formula below: Recovery rate of nucleated cell $=$ (The total cell suspension after separation $\times \mathrm{NC}$ concentration)/(volume of umbilical cord blood before separation $\times \mathrm{NC}$ concentration) $\times 100 \%$. Staining with $4 \%$ trypan blue, and the trypan blue exclusion rate was calculated to measure cell vitality.

\subsection{The Cultivation of Umbilical Cord Blood Cells}

Regulating the concentration of CBMC to $1 \times 10^{6} / \mathrm{ml}$ using PRMI-1640 (GIBCO) culture medium which contained 20\% fetal bovine serum in biosafety cabinet. Then transferring CBMC into a 6-well plate, the capacity of each hole was $35 \mathrm{~mm}$, and only $1 \mathrm{ml}$ CBMC was used for each hole. Repeat 3 times. Cultured for 9 days under the condition of $37^{\circ} \mathrm{C}$, saturated humidity and $5 \% \mathrm{CO}_{2}$ in incubator, and amplified culture twice during this time. Inverted microscope was used to observe cell's growth state. Cells were collected on the ninth day, stained with $4 \%$ trypan blue, and the trypan blue exclusion rate was calculated. 


\subsection{Immunization}

With rubella virus as antigen, stimulating the umbilical cord blood cells which were in well condition with a series concentration of $0.1 \%, 1 \%$ and $10 \%$. Cultured the umbilical cord blood cells for 5 days under the condition of $37^{\circ} \mathrm{C}$, saturated humidity and $5 \% \mathrm{CO}_{2}$ in incubator, and collected the cultured cell supernatant for use.

\subsection{Monoclonal Antibody Purification and IgG Subtype Analysis}

Rubella virus as antigen, indirect ELISA identification was applied to detect and screen those positive umbilical cord blood cells which secreted the monoclonal antibody against rubella virus [12]. Cultured cell supernatant was collected and purified by using Caprylic acid-saturated Ammonium sulfate method [13] [14]. The monoclonal antibody subtype classification kit of sigma was used to do subtype identification [15].

\subsection{Identification of Reactivity and Specificity of Monoclonal Antibody}

In the experimental group, HSV-I, HSV-II, RSV, AS, ADT, CMV, MV and Rubella virus were used as antigen, respectively, and Dot-ELISA were used to identify the reactivity and specificity of Monoclonal antibody [16][19]. Meanwhile, control group should be set. In the control group, HSV-I, HSV-II, RSV, AS, ADT, CMV, MV and Rubella virus were also used as antigen, and the diagnosis of rubella virus monoclonal antibody from traditional way was carried out with Dot-ELISA method. Comparing the experiment results between experimental group with control group.

\section{Results}

\subsection{Cell Viability and Recovery Ratio of Umbilical Cord Blood Cells}

10 copies of umbilical cord blood were collected, and each of the blood volume was $20 \mathrm{ml}-140 \mathrm{ml}$, with (70 \pm $20) \mathrm{ml}$ being average. The CBMC value of each collection, on average, was $(1.21 \pm 0.93) \times 10^{9}$ on a scale from $4.2 \times 10^{8}$ to $4 \times 10^{9}$. The recovery rate of NC was $87.3 \%$, and the trypan blue exclusion rate of recycled NC was greater than $98.9 \%$.

\subsection{Subtype Identification of IgG}

By ELISA, we obtained four strains of positive umbilical cord blood cells. After subculture, cryopreservation and resuscitation in vitro, three of them were confirmed to secrete monoclonal antibody stably, respectively, named MAb 5A2, MAb 2C4, MAb 4E1. The result of monoclonal antibody subtype classification showed that, the antibody secreted by MAb 5A2 and MAb 4E1 belonged to IgG1, while the antibody secreted by MAb 2C4 belonged to IgG2a.

\subsection{Reactivity and Specificity of Monoclonal Antibody}

The experiment result indicated that, in experiment group, none of those antibody secreted by MAb 5A2, MAb 2C4 and MAb 4E1 could specifically reacted with HSV-I, HSV-II, RSV, AS, ADT, CMV or MV, but that all could specifically reacted with rubella virus. The same result goes with the control group which obtained antibody in traditional way by Qingdao University Medical College. This shown that both of the antibody from umbilical cord blood cells and hybridoma cells were specific monoclonal antibody against rubella virus.

\section{Discussions}

At present the general preparation of McAb is through immune experimental animal animal and hybridoma cell to obtain. Experimental animal of the method selection is usually BALB/C mouse, a pure strain of mice, the higher demands on the feeding conditions. The immune status of mouse BALB/C is the premise for hybridoma cells producing antibody, but the serum antibody level of immunized mice was not with the relative relationship. Hybridoma cells is the hybrid cell, in the passage and storage process, the stability of the genome may change, and part of chromosome or gene loss. In addition, the technical requirements of the fusion of B lymphocyte and hybridoma cells for test personnel and the sterile degree of test operation environment are very high. These factors can lead to the preparation of monoclonal antibody to failure, which greatly restricted the preparation and 
application of high quality monoclonal antibody [20].

Because of the ability of more primitive proliferation and more differentiation, umbilical cord blood cells can rebuild long-term hematopoietic stem/progenitor cells. A large number of these cells can be expanded in vitro and its antigen expression is weak, and then the umbilical cord blood cells is expected to replace the experimental animal and hybridoma cell used in the traditional preparation of monoclonal antibody. This study is the first to apply umbilical cord blood cell to the preparation of monoclonal antibody, and make full use of the great resources advantages of umbilical cord blood. Preparing monoclonal antibody with cord blood can make the reduce the cost, short the preparation period, overcome all sorts of factors in the traditional preparation of McAb, and increases the chances of successful preparation of monoclonal antibody. Results reported in this paper has important scientific significance, more inestimable social benefits and economic benefits. This is just a preliminary study that using umbilical cord blood cell to preparation of McAb, and conformed that it is feasibility for preparing monoclonal antibody by umbilical cord blood cell. But it should be further explored whether the same results will also be obtained by other antigen. In addition, the antibody titers, activity, stability and so on also need further study, which umbilical cord blood cells generated after stimulated by antigen.

\section{Conclusion}

This paper has introduced a methodology to preparing monoclonal antibody, and perform that it is feasible for preparing monoclonal antibody by umbilical cord blood cell.

\section{Acknowledgements}

We thank to the Qingdao University School of Medicine Department of Microbiology for providing design tools, support and associated technologies. We are grateful to our department members in Qingdao Zhongren Biotechnology Limited Company R \& D for helpful enthusiastic discussion.

\section{References}

[1] Qian, Z.M., Guo, T.H., Wang, W., Guo, S.Y. and Yuan, Q.M. (2002) The Basic Technology and Clinical Application Research Status of Umbilical Cord Blood. Chinese Journal of Medicine, 41, 568-570.

[2] Rubinstein, P., Carrier, C., Scaradavou, A., Kurtzberg, J., Adamson, J., Migliaccio, A.R., et al. (1998) Outcomes among 562 Recipients of Placental-Blood Transplants from Unrelated Donors. New England Journal of Medicine, 339, 1565-1577. http://dx.doi.org/10.1056/NEJM199811263392201

[3] Gabutti, V., Timeus, F. and Ramenhi, U. (1993) Expansion of Cord Blood Progenitors and Use for Hemopoietic Reconstitution. Stem Cells Dayton, 11, 105-201. http://dx.doi.org/10.1002/stem.5530110818

[4] Chen, J., Sanberg, P.R., Li, Y., Wang, L., Lu, M., Willing, A.E., et al. (2001) Intravenous Administration of Human Umbilical Cord Blood Reduces Behavioral Deficits after Stroke in Rats. Stroke, 32, 2682-2688. http://dx.doi.org/10.1161/hs1101.098367

[5] Sanchez-Ramos, J.R., Song, S., Kamath, S.G., Zigova, T., Willing, A., Cardozo-Pelaez, F., et al. (2001) Expression of Neural Markers in Human Umbilical Cord Blood. Experimental Neurology, 171, 109-115. http://dx.doi.org/10.1006/exnr.2001.7748

[6] Ende, N., Chen, R. and Ende Harris, D. (2001) Human Umbilical Cord Blood Cells Ameliorate Alzheimer's Disease in Transgenic Mice. A Brief Report. Journal of Medicine, 32, 241-247.

[7] Ende, N. and Chen, R. (2001) Human Umbilical Cord Blood Cells Ameliorate Huntington's Disease in Transgenic Mice. A Brief Report. Journal of Medicine, 231-240.

[8] Wang, L.L. and Xu, H.X. (2013) Clinical Immunology Test. 5th Edition, People’s Medical Publishing House, Beijing, $2-11$.

[9] Jin, B.Q. (2008) Medical Immunology. 5th Edition, People’s Medical Publishing House, Beijing.

[10] Zhu, H.P., Sun, Z.M., Wang, J., He, X.D., Dai, H.M., Wu, J.S., et al. (2002) Research on Biological Characteristic of Cord Blood Cell. Journal of Clinical Transfusion and Laboratory Medicine, 2, 8-10.

[11] Liu, G.J., Guo, L.R. and Qiao, Z.H. (2002) Study on Biological Characteristic of Umbilical Cord Blood Cells. Shanxi Clinical Medicine Journal, 11, 406-410.

[12] Huang, L.P., Liu, C.M., Wei, Y.W., Zhang, Z.X., Lu, Y.H. and Guo, L.J. (2009) Preparation and Identification of Neutralizating Monoclonal Antibody to the Recombinant Capsid Protein of Porcine Circovirus Type 2. Chinese Journal of Preventive Veterinary Medicine, 31, 132-136. 
[13] Chen, D., Sun, G.R. and Liu, Z.S. (2007) Application of Caprylic Acid Ammonium Sulfate Precipitation Method Incombination of Monoclonal Antibody Purification. Journal of Anhui Agricultural Sciences, 35, 8105-8108.

[14] Zhou, Y., Li, Y.S., Pan, F.G., Tan, J.H., Liu, Z.S. and Wang, Z. (2006) The Study of Purified Methods of Mice Ascites IgG McAb. Heilongjiang Animal Science and Veterinary Medicine, 10, 14-16.

[15] Dong, G.H., Wei, P., Huang, J.Q., Du, Q.B., Yue, H. and Du, W.S. (2014) IL-31 Establishment of ELISA Method for Detection of IL-31 and Preparation of the Monoclonal Antibody. Chinese Journal of Immunology, 4, 495-498.

[16] Ti, J.F., Li, Z.J. and Li, F. (2012) The Isolation and Identification of Duck Hepatitis Virus I of Shandong Area and Development of Its Monoclonal Antibody. Progress in Veterinary Medicine, 33, 63-66.

[17] Pan, P.P., Wen, L.Z., Shan, Z.Z., Ding, Y., Yuan, B. and Ren, W.S. (2011) Monoclonal Antibody against Duck Hepatitis Virus Preparation. Chinese Agricultural Science Bulletin, 27, 415-441.

[18] Wang, W., Wang, X.M., Wen, L.B., He, K.W., Zhou, J.M., Guo, R.L., et al. (2014) Preparation and Identification of Specific Monoclonal Antibody against Porcine Circovirus Type 2. Agricultural Science and Technology, 15, 173-176.

[19] Zhang, L., Wang, Y.Q., Hao, L.S., Gao, M.M., Li, A.D. and Tao, Y. (2014) Preparation of the Monoclonal Antibody against Cap Protein of Porcine Circovrius typ2 and Its Antigenic Epitope Identification. Chinese Journal of Preventive Veterinary Medicine, 4, 310-313.

[20] Qi, X. (1999) The Preparation of the Monoclonal Antibody of Rubella Virus. Journal of Lanzhou Medical College, 25, 10-11. 